Compte rendu de thèse

\title{
Alexandre Tessier, Le Grand Hôtel, 110 ans d'hôtellerie parisienne, 1862-1972
}

Thèse de doctorat en histoire, université François-Rabelais (Tours), dirigée par Marc de Ferrière Le Vayer (soutenue le 24 novembre 2009)

\section{Laurent Tissot}

\section{OpenEdition Journals}

Édition électronique

URL : http://journals.openedition.org/tourisme/463

DOI : 10.4000/tourisme.463

ISSN : 2492-7503

\section{Éditeur}

Éditions touristiques européennes

\section{Édition imprimée}

Date de publication : 1 décembre 2011

Pagination : $92-94$

ISSN : 2109-5671

\section{Référence électronique}

Laurent Tissot, "Alexandre Tessier, Le Grand Hôtel, 110 ans d'hôtellerie parisienne, 1862-1972 », Mondes du Tourisme [En ligne], 4 | 2011, mis en ligne le 30 septembre 2015, consulté le 22 septembre 2020. URL : http://journals.openedition.org/tourisme/463 ; DOI : https://doi.org/10.4000/tourisme.463

\section{(c) (i) (9)}

Mondes du tourisme est mis à disposition selon les termes de la licence Creative Commons Attribution - Pas d'Utilisation Commerciale - Pas de Modification 4.0 International. 


\title{
COMPTE RENDU DE THËSE (PAR LAURENT TISSOT)
}

\section{Le Grand Hôtel, I I 0 ans d'hôtellerie parisienne, I862-I972}

\author{
Thèse de doctorat en histoire, université François-Rabelais (Tours) \\ dirigée par Marc de Ferrière Le Vayer \\ présentée par A LEXANDRE TESSIER (soutenue le 24 novembre 2009)
}

$\mathbf{S}^{\prime}$ il est un secteur d'activité qui reste encore en friche dans le domaine de l'histoire économique et sociale, c'est bien l'hôtellerie. Le bilan historiographique est d'une maigreur incompréhensible compte tenu de sa place dans le développement économique et social de ces cent cinquante dernières années. C'est dire si la thèse d'Alexandre Tessier vient à son heure. C'est dire aussi si elle est attendue et, disons-le tout de suite, elle ne déçoit pas. Forte de 783 pages, dont 633 pages de texte principal, elle brosse un tableau complet, sous la forme d'une monographie d'entreprise, d'un hôtel ayant joué un rôle central dans la "construction" du Paris touristique et hôtelier. À la fois exemple et modèle, le Grand Hôtel méritait une telle attention parce qu'il réunit les signes d'élection de la grande hôtellerie qui s'impose dès le milieu du XIX ${ }^{e}$ siècle sur le continent européen, mais aussi sous d'autres latitudes. C'est dire aussi que, sans points de repère forts, le travail ne se présentait pas sans risque.

En s'attelant à faire l'histoire du Grand Hôtel, l'auteur s'est heurté aux difficultés qui justement expliquent les raisons pour lesquelles l'hôtellerie ait si peu retenu l'attention : l'existence d'archives substantielles et leur accès. Alexandre Tessier peut se classer parmi les historiens "heureux" en la matière tant les archives du Grand Hôtel sont d'une qualité tout à fait remarquable et sur les matières traitées et sur la période considérée, mais ces archives souffrent aussi de lacunes inhérentes à l'histoire des entreprises, lacunes qu'il a comblées grâce à un sens de la curiosité, et aussi à une grande imagination qui devrait être le lot de tout historien. Le recours aux archives nationales, aux archives départementales, aux archives de la préfecture de police, aux archives militaires, à la Bibliothèque historique de la ville de Paris, aux archives du Crédit Agricole, à des archives privées ainsi qu'à une vaste littérature de sources imprimées, parmi lesquelles il faut signaler l'abondante littérature sur les restaurants, les cafés et hôtels, témoigne de la capacité d'Alexandre Tessier de réunir une vaste documentation, de l'organiser et de l'exploiter en fonction des thématiques développées.

Un autre risque résidait dans le manque de repères problématiques : Pourquoi faire une histoire d'un hôtel ? Que peut-elle apporter à notre connaissance de nos sociétés ? L'auteur décrit les pistes qu'il entend explorer : "[...] Comprendre comment le Grand Hôtel fut géré [...], quels furent les choix retenus par ses divers dirigeants [...] pouvant expliquer sa longévité" (p. 13). L'intégration de la politique sociale, 
du recrutement, de la gestion du personnel sont aussi des points abordés. En prenant encore l'option de s'atteler à une histoire longue (cent dix ans) de l'entreprise, il a pris résolument la voie d'une grande ambition, celle d'envisager une véritable histoire "totale" du Grand Hôtel, sans forcément bénéficier des repères fondamentaux qui auraient pu lui faciliter la tâche. Si le propre d'une thèse est d'être ambitieuse, ce trait doit s'inscrire dans une réflexion méthodologique et problématique, même pour un champ scientifique naissant. À force de vouloir tout dire, on ne dit plus rien.

Le recoupement des sources et leur mise en perspective débouchent sur des éclairages très novateurs sur les différents modes de fonctionnement qui visèrent à faire du Grand Hôtel une entreprise de grande envergure compte tenu des contraintes locales et des ambitions déclarées. Le souci d'être toujours proche des acteurs assure aux observations d'Alexandre Tessier une très haute qualité de démonstration, reposant sur un rapport à l'archive toujours bien mise en contexte et clairement décrite. Le travail est rédigé dans une langue élégante, sans fioritures ni effets de manche et qui a toujours pour but d'aller à l'essentiel sans pour autant dénaturer la complexité des démarches analysées et la variété des situations évoquées. Il est mené avec une redoutable efficacité, qui traduit toute la détermination du candidat à surmonter les nom- breuses difficultés auxquelles ce genre de travail peut se heurter. La très grande qualité des annexes, autant celle des tableaux synthétiques et des cartes que celle des photographies, souligne la richesse des matériaux utilisés et leur potentialité démonstrative.

S'appuyant sur une approche chronologique, le travail est divisé en trois parties qui coïncident avec les époques principales du Grand Hôtel. Dans la première partie Naissance d'un géant, 1860-1879 -, Alexandre Tessier s'attache à montrer la création du Grand Hôtel, son insertion dans le tissu urbain parisien, son développement qui suit les premières années ainsi que sa signification sur les plans économique, social et symbolique. La mise en exergue de l'action des frères Pereire est à cet égard très intéressante, parce qu'elle montre l'imbrication des activités d'entrepreneurs dont seuls les aspects financiers et ferroviaires avaient jusqu'ici été soulignés. Dans la deuxième partie - Entre reprise et fonctionnement interne, 18791898 -, il offre une analyse très précise du fonctionnement de l'hôtel grâce à des archives particulièrement complètes sur cette période. L'intervention de la Banque parisienne et son dessaisissement, la gestion financière et commerciale ainsi que la politique de communication sont les aspects développés. La troisième partie - La dynastie Millon, 1898-1972 - est consacrée à la prise en main du Grand Hôtel par Arthur Millon, qui donne à l'entreprise une forte prégnance familiale. La perte de contrôle familial en 1972 marque une très bonne césure dans l'analyse, même si l'histoire du Grand Hôtel se poursuit...

Alexandre Tessier démontre avec beaucoup de conviction la pertinence de ce canevas. Il suit une analyse rigoureuse et systématique fondée sur une exploitation fine et approfondie des multiples sources utilisées, une érudition des situations évoquées et une curiosité de très bon aloi sans pédantisme aucun.

Sur le fond, les qualités de ce travail sont de plusieurs ordres. Elles s'accompagnent néanmoins d'un certain nombre de faiblesses que nous mentionnons en cours de rédaction, en émettant certain nombre de critiques "constructives" qui sont de nature à aider Alexandre Tessier dans un travail de publication que l'on souhaite de tous nos vœux.

1) L'histoire du Grand Hôtel qui nous est présentée souligne toute l'épaisseur de cette activité. L'auteur insère son objet dans un contexte large. Une activité hôtelière ne s'arrête pas à l'exploitation d'un hôtel. Elle déborde de son immeuble. C'est un ensemble d'activités qui est en jeu, qui regroupe au sens large du terme le logement, la restauration, le commerce, la distraction, la sociabilité et dont les impacts sont très grands en termes de reconfiguration de l'espace urbain, de l'espace social, de l'espace économique, de l'espace 
professionnel, etc. En s'intéressant à tout ce qui "tourne" autour de l'hôtel et qui le fait véritablement vivre, Alexandre Tessier nous montre ce qui fait finalement exister un hôtel - des boutiques, des cafés, des services multiples et divers (communication, transports, théâtre...) - autant que les chambres qu'il loue et les repas qu'il propose. Dans cette perspective, il redonne au genre monographique une place essentielle dans l'approche économique en lui joignant une dimension "multifonctionnelle" et "multidimensionnelle" à très haute valeur ajoutée.

2) Complémentaire au premier point, la spécificité de l'activité hôtelière est aussi bien mise en évidence par Alexandre Tessier. Son étude montre le soin qu'il faut porter à la description d'une activité économique quelle qu'elle soit, aux produits qu'elle entend vendre et aux marchés qu'elle vise. L'activité hôtelière n'est pas comparable à d'autres activités, parce qu'elle s'exerce sur un terrain extrêmement délicat qui réunit différentes compétences et différents services. En alliant une étude de la partie visible et spectaculaire exposée aux regards du public et du client (la conception du bâtiment, la disposition des chambres, l'ornementation architecturale, la situation de l'édifice, etc.) à celle de la partie moins visible, et même pour beaucoup invisible (les cuisines, les caves, la machinerie, les dépôts, le ravitaillement, etc.), Alexandre Tessier en dévoile toute la com- plexité. Par là même, il nous révèle aussi toute la complexité du métier d'hôtelier, qui consiste à faire jouer ensemble tous ces éléments de telle sorte qu'ils s'imbriquent le plus harmonieusement possible. Nous avons beaucoup aimé les pages consacrées aux cuisines, à l'ascenseur, aux chambres, au mobilier, qui donnent véritablement toute la substance de l'activité hôtelière. Dans cette perspective, nous aurions aimé qu'il attache plus d'attention aux différents directeurs qui ont travaillé au Grand Hôtel. Décrire les mécanismes qui amènent des administrateurs à opérer des coups financiers par des (r)achats et des ventes de toutes sortes est nécessaire, mais la capacité d'un hôtel à survivre passe par une gestion quotidienne que l'on aurait aimé voir plus présente et plus précise. Quelques allusions sont données (p. 469), mais sans plus, alors que le directeur est au cœur de tout. La professionnalisation du métier d'hôtelier ne se réduit pas ainsi à savoir prendre la parole dans un conseil d'administration et à faire jouer des réseaux, à manipuler les budgets et distribuer des dividendes. Elle se reconnaît surtout dans le savoirfaire, l'aptitude à organiser cet univers aussi divers et spécialisé. C'est principalement au travers du personnage du directeur que s'opère véritablement une rapide internationalisation qui caractérise la branche et qui lui donne, à partir de 1880, une entité bien précise et un cursus bien délimité. La création d'écoles hôtelières est à cet égard la marque principale d'une véritable assise d'une profession capable dès lors de formaliser les faits et gestes, de répertorier toutes les fonctions et d'évaluer les compétences requises. Si "la gestion quotidienne $d u$ Grand Hôtel devient de plus en plus rigoureuse" (p. 474), c'est qu'elle traduit le fort impact des modèles transnationaux qui fondent la grande hôtellerie. Les pages consacrées à Arthur Millon sont importantes et utiles (p. 382 et suiv.), mais elles auraient mérité une meilleure insertion dans cette multinationalisation de la grande hôtellerie, dont Alexandre Tessier nous avance certains éléments (la présence allemande, suisse ou américaine) sans nous en donner une claire image. L'histoire du Grand Hôtel dépasse le cadre - important - parisien de son insertion pour toucher à l'émergence de modèles internationaux.

Au total, Alexandre Tessier nous présente une thèse bien documentée, très solide et plaisante à lire, qui mérite pleinement d'être défendue car elle éclaire de manière très neuve la question essentielle de l'hôtellerie, sans laquelle il est vain de prétendre aborder, d'une façon intelligible, non seulement l'histoire des services, mais l'histoire économique de la France et de l'Europe. Ce faisant, il nous propose une approche très éclairante de cette question.

Laurent Tissot 